\title{
Does periodontal disease affect pregnancy outcome?
}

\author{
A prospective study to investigate the relationship between periodontal disease and adverse pregnancy outcome \\ S. Moore, M. Ide, P. Y. Coward, M. Randhawa, E. Borkowska, R. Baylis and R. F. Wilson
}

\section{Objective}

This study aimed to investigate a relationship between maternal periodontal disease and preterm birth, low birth weight and late miscarriage.

\section{Design}

Prospective study in single centre.

\section{Setting}

Guy's and St Thomas' Hospital Trust, London, UK between August 1998 and July 2001.

\section{Subjects and methods}

Pregnant women were recruited on attending an ultrasound scan at approximately 12 weeks of pregnancy. Subjects completed a questionnaire and underwent periodontal examination.

\section{Main outcome measures}

Plaque and bleeding scores, pocket probing depth and loss of attachment. Pregnancy outcome data was collected retrospectively, including gestational age and birth weight at delivery.

\section{Results}

Data were collected for 3,738 subjects. Regression analysis indicated that there were no significant relationships between the severity of periodontal disease and either preterm birth (PTB) or low birth weight (LBW). In contrast, there did appear to be a correlation between poorer periodontal health and those that experienced a late miscarriage.

\section{Conclusions}

There was no association between either preterm birth or low birth weight and periodontal disease in this population. There is evidence of a correlation between markers of poorer periodontal health and late miscarriage.

\section{IN BRIEF}

- This study has a more representative sample of pregnant women in the UK when compared with previous studies investigating associations between periodontal disease and adverse pregnancy outcome.

- Periodontal disease did not appear to be a risk factor for premature or low birth weight birth in their population.

- Periodontal disease does appear to be associated with miscarriage between 12 and 24 weeks gestation, even after controlling for other risk factors of poor pregnancy outcome. It is not yet known whether treating periodontal disease in pregnancy can reduce the risk of miscarriage.

\section{COMMENT}

Adverse pregnancy outcome is multi-factorial and risk factors include child bearing age, racial origin, poverty, maternal education, smoking, malnutrition, multiple gestation, previous poor obstetric outcome and genito-urinary infection. In recent years periodontal disease has been cited as an additional risk factor for the delivery of preterm and low birth weight infants. This paper reports the second study conducted in the United Kingdom, the first being carried out in the East End of London. ${ }^{1}$ Likewise, it fails to find an association between maternal periodontal disease in the first trimester of pregnancy and preterm or low birth weight birth. However, there seems to be a weak relationship between poor periodontal health and late miscarriage.

This study involved 3,823 subjects who presented for a nuchal translucency scan for Down's Syndrome at around 12 weeks of pregnancy, at a South London NHS Trust and followed until delivery. A total of $286(7.7 \%)$ subjects had preterm birth (less than 37 weeks gestation), and 49 had a late miscarriage (between 12 and 24 weeks gestation) or intrauterine death at 24 weeks gestation. The data collection included a wide range of risk factors associated with both periodontal disease and adverse pregnancy outcomes. A periodontal examination included a full mouth, two sites per tooth assessment for the presence of plaque, probing depth $(\mathrm{mm})$, loss of periodontal attachment $(\mathrm{mm})$, and bleeding on probing was carried out. No treatment was given to the subjects; however an oral hygiene pack was issued.

The main findings were insignificant in terms of periodontal disease, except for those women who had a late miscarriage where the mean probing depth, median loss of attachment was increased, as well as a higher proportion of sites probing $4 \mathrm{~mm}$ or greater. The level of periodontal disease was not dissimilar to the general UK population age group. The study revealed that classic obstetric risk factors such as ethnicity, socioeconomic status, medication and previous poor obstetric outcome were associated with preterm birth at less than 37 weeks gestation and low birth weight of less than $2,500 \mathrm{~g}$.

The authors discussed the limitation of their study especially the use of self reported data not least that smoking may be inaccurate, the method of recording periodontal variables which were kept to the minimum to keep the examination time and discomfort to a minimum to ensure maximum recruitment, data collection carried out by midwives on busy maternity wards and the number of analyses carried out all of which may be partly instrumental in the lack of relationship. As Davenport and colleagues ${ }^{1}$ discussed, the main differences are most likely to be accounted for by differences in populations in terms of periodontal health and incidence of poor pregnancy outcome.

This large study has provided further evidence that maternal periodontal disease is not a risk factor in the delivery of preterm or low birth weight infants in the UK. However, it does not preclude the need to maintain good oral health during pregnancy.

1. Davenport ES, Williams C E CS, Sterne J A C, Murad S, Sivapathasundram V, Curtis M A. Maternal periodontal disease and preterm low birthweight: case-control study. J Dent Res 2002: 81; 313-318.

E. S. Davenport, Professor of Dental Education, Barts and The London Queen Mary's School of Medicine and Dentistry, doi:10.1038/sj.bdj.4811619 